\title{
About the origin of low wafer performance and crystal defect generation on seed-cast growth of industrial mono-like silicon ingots
}

\author{
Ismael Guerrero, Vicente Parra *, Teresa Carballo , Andrés Black, Miguel Miranda , \\ David Cancillo , Benito Moralejo , Juan Jiménez , Jean-François Lelièvre- and Carlos del Cañizo
}

\begin{abstract}
The era of the seed-cast grown monocrystalline-based silicon ingots is coming. Mono-like, pseudomono or quasimono wafers are product labels that can be nowadays found in the market, as a critical innovation for the photovoltaic industry. They integrate some of the most favorable features of the conventional silicon substrates for solar cells, so far, such as the high solar cell efficiency offered by the monocrystalline Czochralski-Si (Cz-Si) wafers and the lower cost, high productivity and full square-shape that characterize the well-known multicrystalline casting growth method. Nevertheless, this innovative crystal growth approach still faces a number of mass scale problems that need to be resolved, in order to gain a deep, $100 \%$ reliable and worldwide market: (i) extended defects formation during the growth process; (ii) optimization of the seed recycling; and (iii) parts of the ingots giving low solar cells performance, which directly affect the production costs and yield of this approach. Therefore, this paper presents a series of casting crystal growth experiments and characterization studies from ingots, wafers and cells manufactured in an industrial approach, showing the main sources of crystal defect formation, impurity enrichment and potential consequences at solar cell level. The previously mentioned technological drawbacks are directly addressed, proposing industrial actions to pave the way of this new wafer technology to high efficiency solar cells. Copyright $\odot 2012$ John Wiley \& Sons, Ltd.
\end{abstract}

KEYWORDS

casting; mono-like; silicon wafer; photoluminescence; dislocation; LBIC

\section{INTRODUCTION}

Solar market and technology are largely based on crystalline silicon. Typically, two solar cell technologies can be developed according to the crystal features of the silicon substrates used: monocrystalline, by the well-known Czochralski growth method (Cz-Si) and multicrystalline (mc-Si), usually based on casting techniques (using Bridgman-type furnaces). Cz-Si ingots imply higher solar cell efficiency because of the optimized mono surface texturing and lower amount of crystal defects, such as grain boundaries and dislocations, with respect to mc-Si materials. Higher oxygen concentrations are also expected, which can result in light-induced degradation patterns when considering Boron-doped materials [1]. In contrast, the manufacturing of mc-Si materials is relatively straightforward and more cost-effective. Also, $\mathrm{Cz}-\mathrm{Si}$ wafers are usually presented in the market as pseudo-square substrates, whereas mc-Si is full squareshaped substrate.

Nowadays, the unclear and volatile photovoltaic (PV) market demands highly innovative approaches that can reduce the production costs of the materials and devices, but simultaneously increasing the energy $/ \mathrm{m}^{2}$ ratio. In particular, one of the most attractive approaches in the PV field so far, the brand-new mono-like wafers, are entering the market as one of the most promising advances. These wafers present monocrystalline features on ingots 
manufactured according to commercial cast growth furnaces $[2,3]$. As a result, the optimum wafer substrates share some of the main advantages of the well-established $\mathrm{Cz}$-Si mono crystals and the cost-effectiveness and mass scale issues of mc-Si. These novel wafers are supposed to offer up to $1.0-1.5 \%$ extra efficiency with respect to the usual mc-Si substrates, depending on the wafer texturing process (alkaline-anisotropic or acid-isotropic) being applied.

However, significant problems can be detected when analyzing an ingot manufactured according to the worldwide extended industrial casting standards, as high generation of bulk extended defects, unusual contamination phenomena by residual impurities and the appearance of considerable extended multicrystalline grains regions in the ingot. In addition, mass scale production costs increase, as the monocrystalline seeds reutilizaton is neither evident nor straightforward. Low solar cell efficiency ingot regions directly associated with low carrier lifetime regions of the mono-like ingot are present; consequently, the expected high wafer performance and yield can be seriously suppressed. The literature dealing with defect formation in seed-cast growth is scarce, specially at lab scale background [4]. Numerical simulations addressing some of the previously mentioned issues have also been recently published, by Gao et al. $[5,6]$.

We review, in this paper, a detailed and critical analysis of the most deleterious sources of low wafer performance and crystal defect generation during the seed-cast growth approach based on a series of experiments performed in a full industrial environment (ingot, wafer and solar cell manufacturing). Both silicon materials and devices have been characterized using several optoelectronic techniques, such as photoluminiscence (PL) imaging, microwave photoconductance decay ( $\mu$-PCD) and high resolution laser beam induced current (LBIC). Finally, practical ideas to manage the technical limitations imposed by this attractive crystal growth process are also proposed, to pave the way of this necessary innovation to the PV technology at the very short-term.

\section{EXPERIMENTAL SECTION}

\subsection{Ingot manufacturing and characterization techniques}

Seed-cast growths were performed at DC Wafers Investments, S.L. Spain, using both Directional Solidification (DSS) and Heat Exchange Method (HEM) furnace stations (from GT Solar, USA). Cz-Si ingots for seed manufacturing were produced at CENTESIL Institute, in Madrid (Spain), using a PVA-Tepla $\mathrm{Cz}$ puller. The crystal growth processes were labeled according to the number of repeated uses of the original $\mathrm{Cz}$ seed (ingot growth runs G0, Gn, Gn + 1, etc.), suitably cropped from the silicon bricks after each growth process. Different monocrystalline seed configurations were tested, from $1-(1 \times 1)$ to $25-(5 \times 5)$ silicon slabs.
Bulk carrier lifetime of silicon bricks was measured by a STV Telecom $\mu$-PCD instrument (Russia). For the wafer characterization, PL images over the whole wafer surface were collected by a PLI-101 Inline Photoluminescence Imaging System. Minority carrier lifetime analysis was also performed on as-cut wafers, by both the Quasi Steady-State Photoconductance (QSSPC WCT-120, Sinton Instruments, Boulder, CO, USA) and $\mu$-PCD mappings (Semilab WT 2000). The wafers were chemically etched to remove the saw damage, then coated by SiNx:H at both sides. Interstitial iron concentration $\left(\left[\mathrm{Fe}_{\mathrm{i}}\right]\right)$ was also measured, using the $\mathrm{Fe}-\mathrm{B}$ dissociation technique. An additional phosphorus gettering process (standard $\mathrm{POCl}_{3}$-based process) was implemented to analyze the evolution of $\left[\mathrm{Fe}_{\mathrm{i}}\right]$ in the samples.

A homemade LBIC system operating with three excitation wavelengths, from two dual laser diodes (Omicron) with $639-830 \mathrm{~nm}$ and $853 \mathrm{~nm}$ laser lines, besides a second laser diode $(785 \mathrm{~nm})$ was used. A beam splitter directs the laser beam into a trinocular microscope, which is used to focus the excitation laser beam onto the sample surface. Three signals are acquired, stored simultaneously, and correlated to the sample $(x, y)$ coordinates to building up the LBIC maps: (i) the output power of the excitation laser, (ii) the light power reflected by the wafer; and (iii) the electric current induced by the laser beam (LBIC). Microscope objectives with different magnifications and numerical apertures were used, allowing the acquisition of high spatial resolution LBIC images. The sample is mounted on a motorized translation stage with three stepping motors (XYZ motion) manufactured by Prior Scientific, Cambridge, UK. The software of control acquisition and data treatment was developed under Labview v 8.5. [7].

Solar cells $(156 \mathrm{~mm} \times 156 \mathrm{~mm})$ from mono-like wafers were produced by using two different methods: (i) typical acid texturing processes, $\mathrm{POCl}_{3}$ diffusion and an $\mathrm{H}$-patterned screen-printing metallization (average mc-Si cell efficiency $16.4 \%$ ); and (ii) alkaline texturing followed by ion implantation emitter formation, without implementing any selective emitter technology.

\subsection{Crucibles and seeds}

Gen 5 RTU quartz crucibles (Vesuvius, France) were used to manufacture the ingots, which were based on solar grade polysilicon.

P-type and non-doped $\mathrm{Cz}-\mathrm{Si}<100\rangle$ oriented monocrystalline seeds $(14.0 \mathrm{~cm} \times 14.0 \mathrm{~cm})$ were used for the seed-cast growth experiments. Silicon seed thickness varied from 10 to $30 \mathrm{~mm}$.

\section{RESULTS AND DISCUSSION}

\subsection{Industrial manufacturing of mono-like ingots. Experimental facts to consider}

A seed-cast growth technique has been successfully implemented to manufacture industrial ingots having clear 
monocrystalline features. Figure 1 shows a transverse section of a $450 \mathrm{~kg}$ DSS ingot, showing a large monocrystallinefashioned volume all along the bulk. Some mc-Si regions are also observed at the edges of the ingot, as indicated by arrows in the figure. The wafers sliced from such an ingot are ranged in different mono-like classes, according to the size of their respective monocrystalline sections (from $100 \%$ monocrystalline to less than $75 \%$, in the

a)
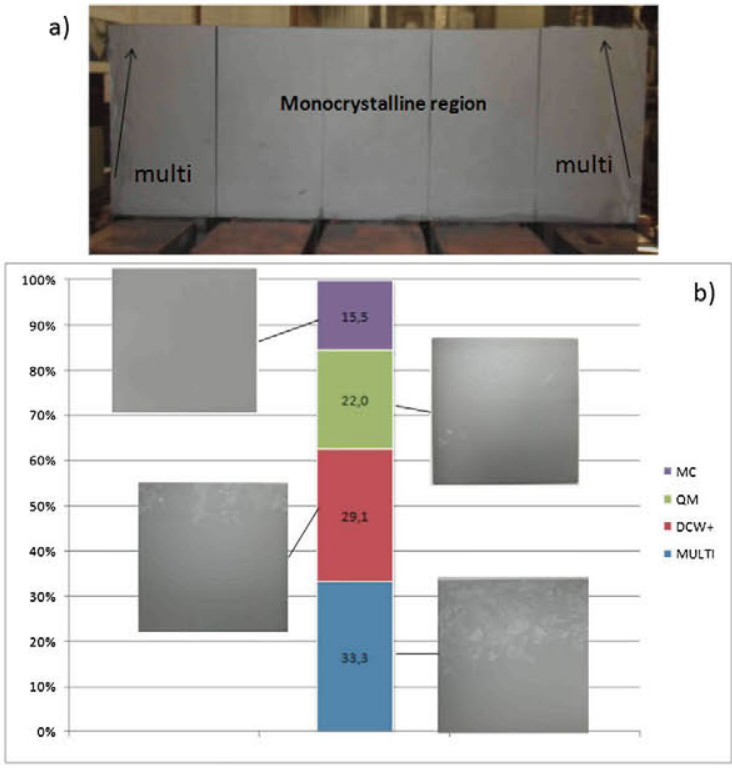

Figure 1. (a) Picture of a typical Go mono-like ingot manufactured at DC Wafers by implementing the seed-cast growth method (DSS method); (b) Wafer-type distribution according to their percentage of macroscopic monocrystalline feature. $\mathrm{MC}$, monocasting $(100 \%)$; QM, quasimono (>90\%); DCW+, DC Wafers PLUS (>75\%); MULTI, mono/multi and mc-Si $(<75 \%)$. present case). In particular, a high percentage of full square monocrystalline wafers $(15.6 \mathrm{~cm} \times 15.6 \mathrm{~cm})$ can be obtained in a straightforward way, even when using a commercial casting furnace technology.

However, in a G0 crystal growth process, on the basis of virgin monocrystalline seeds from a $\mathrm{Cz}$ ingot, both the proportion of mono-like wafers and their intrinsic defect density, from the center to the sides and from tail to top, strongly depend on a number of experimental variables, which need to be designed and optimized to avoid solar cell performance loses. The seed-cast growth method is based on the use of perfectly oriented monocrystalline square silicon slabs, usually $\langle 100\rangle$, which are properly placed at the bottom of the quartz crucible [3]. The crystal melt/growth process is consequently based on the application of a passive cooling from the bottom of the crucible to geometrically control the melting of the seed up to a defined height, using a calibrated quartz rod, then applying a corresponding cooling before the seed disappears into the melt. As a result, the crystal orientation of the seed is transferred to the top of the ingot. This potentially tricky task can be achieved either by designing and implementing optimized furnace setting parameters (software approach) and/or by adapting the graphite hot zone in the proper way (hardware approach).

The mere fact of placing the seeds into the crucible, besides the quality of their respective edges resulting from the sawing process, can promote the generation of crystal defects in an industrial mono-like ingot (G0, G1, etc.). This is illustrated in Figure 2, in which two different types of seed configurations were tested on the basis of both multi and monocrystalline materials, to check how the junctions between adjacent seeds can act as sources of extended crystal defects.

Despite the full monocrystalline aspect of the wafers obtained from the silicon brick obtained using a $2 \times 2$ central

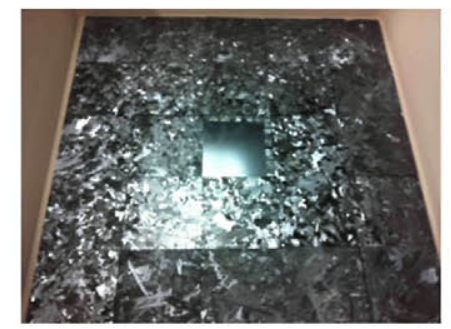

\section{Side growth of multicrystals}

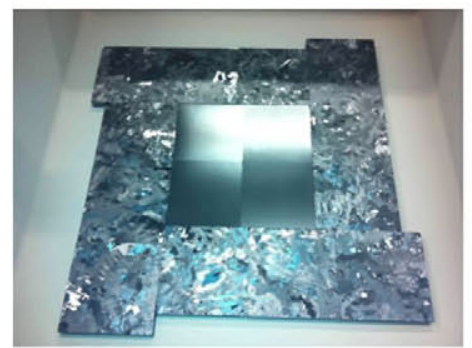

Seed junctions

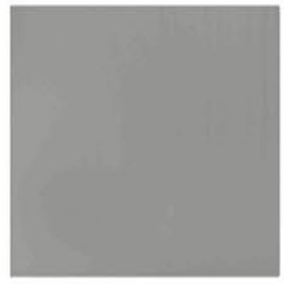

Figure 2. Different seed placing schemes to detect potential sources of defects from the very beginning of the seed-cast growth $(1 \times 1$ centered and $2 \times 2$ seed configurations, respectively). 
seed configuration, an analysis of the current-voltage $(I-V)$ solar cell parameters showed large deviations in the absolute efficiency values, exhibiting undesirable low-performance efficiency tails, particularly for wafers arising from the top section of the brick. Such a broadly dispersed electrical performance was also observed in the mono/multi-based brick, resulting however in lower absolute efficiencies by $0.5 \%$, due to the mc-Si side growth. In wafers from a full monocrystalline seed configuration $(5 \times 5$ seed design $)$, the $I-V$ performance was found to exhibit double peak distribution for both short circuit current $\left(I_{\mathrm{se}}\right)$ and open circuit voltage $\left(V_{\text {oc }}\right)$, which resulted in a double peaked efficiency distribution (Figure 3(a)). Interestingly, such a detrimental outcome was markedly observed in wafers having large mono-like regions, as MC (100\% surface) and QM (>90\% surface) series. This two-peaked electrical performance distribution was also observed in solar cells made from wafers from an MC series brick (table included in Figure 3(b)), but processed using alkaline texturing processes (plus ion implantation P-doping). This confirms the large performance distributions that can be obtained from these industrially cast monocrystalline ingots, even considering the same brick, based on complete monocrystalline wafers.

Crystal growth issues were suggested as the main origin of such a remarkable double peak distribution of the electrical parameters. Thus, an optoelectronic study was conducted, for which both PL and $\mu$-PCD minority carrier lifetime analysis over the whole surface of a series of wafers representing the bottom (above the seed region interface) and top sections of the brick were performed. The PL analysis of Figure 4 allowed to conclude that an undesirable propagation of extended defects from bottom to top clearly occurred (similar to what is typically observed in mc-Si cast growth), which seems to be favored by the seeds junctions at the very beginning of the growth run. The $\mu$-PCD lifetime analysis revealed exceptionally high $\mathrm{Fe}$ interstitial concentration $\left[\mathrm{Fe}_{\mathrm{i}}\right]$ at the bottom region of the bricks (ca. $5.3 \times 10^{12} \mathrm{~cm}^{-3}$ ), decreasing by more than one order of magnitude (ca. $1.4 \times 10^{11} \mathrm{~cm}^{-3}$ ) when going from bottom to top (Figure 4), regardless of the low segregation coefficient of this element in silicon $\left(2 \times 10^{-5}\right)$. Therefore, this would indicate that the efficiency decrease of solar cells made of wafers sliced from the top sections of the ingot is mostly dominated by the defect generation during the crystal growth. Indeed, it is well-known that the distribution of the Fe contamination, mostly entering the melt by solid-state diffusion from the crucible and silicon nitride coating, tends to move from interstitial to precipitated $[8,9]$, from bottom to top of the ingot. Thus, in order to investigate this effect on mono-like wafers, Figure 5(a) illustrates the evolution of $\left[\mathrm{Fe}_{\mathrm{i}}\right]$ before and after a typical phosphorus gettering (PDG) process.

It can be observed how the initial $\left[\mathrm{Fe}_{\mathrm{i}}\right]$ concentrations are differently reduced after implementing the same PDG process. The gettering effect is markedly observed in the case of bottom wafers $\left(\left[\mathrm{Fe}_{\mathrm{i}}\right]\right.$ decrease in more than two orders of magnitude), which demonstrates that most of the Fe present in the crystal is presented under a dissolved state. In contrast, the effect of the defect generation towards the top results in lower $\left[\mathrm{Fe}_{\mathrm{i}}\right]$ reduction, which suggests that $\mathrm{Fe}$ can move to precipitation sites, probably the defective regions observed by PL mappings.

This consistency between low lifetime and high $\mathrm{Fe}$ concentration at the bottom zone of the brick is directly attributed to the competition between the rate of Fe segregation just after the crystal nucleation and the back-diffusion of Fe coming from the crucible walls and coating just before the melting phase end [5]. This effect results in both seed degradation and pronounced low lifetime tails above the seed,

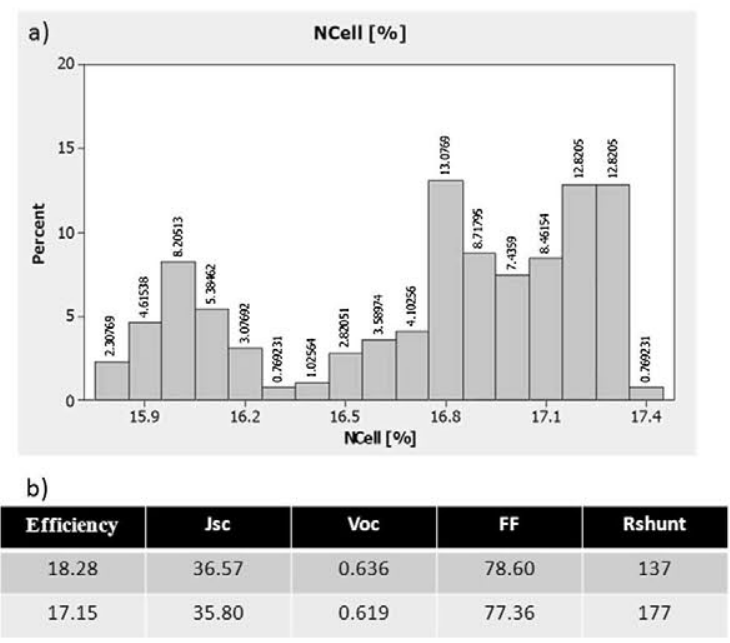

Figure 3. (a) Typical efficiency distribution from a seed-cast ingot for $M C$ and $\mathrm{QM}$ wafer series processing. The typical efficiency average value for common mc-Si wafers, using the same solar cell methods is $16.4 \%$; (b) $1-V$ values obtained using alkaline texturing and ion implantation phosphorus doping (without any selective emitter implementation), in the case of MC wafers from the same brick. The values represent the average parameters resulting from the two-peaked solar cell distribution that was observed. 

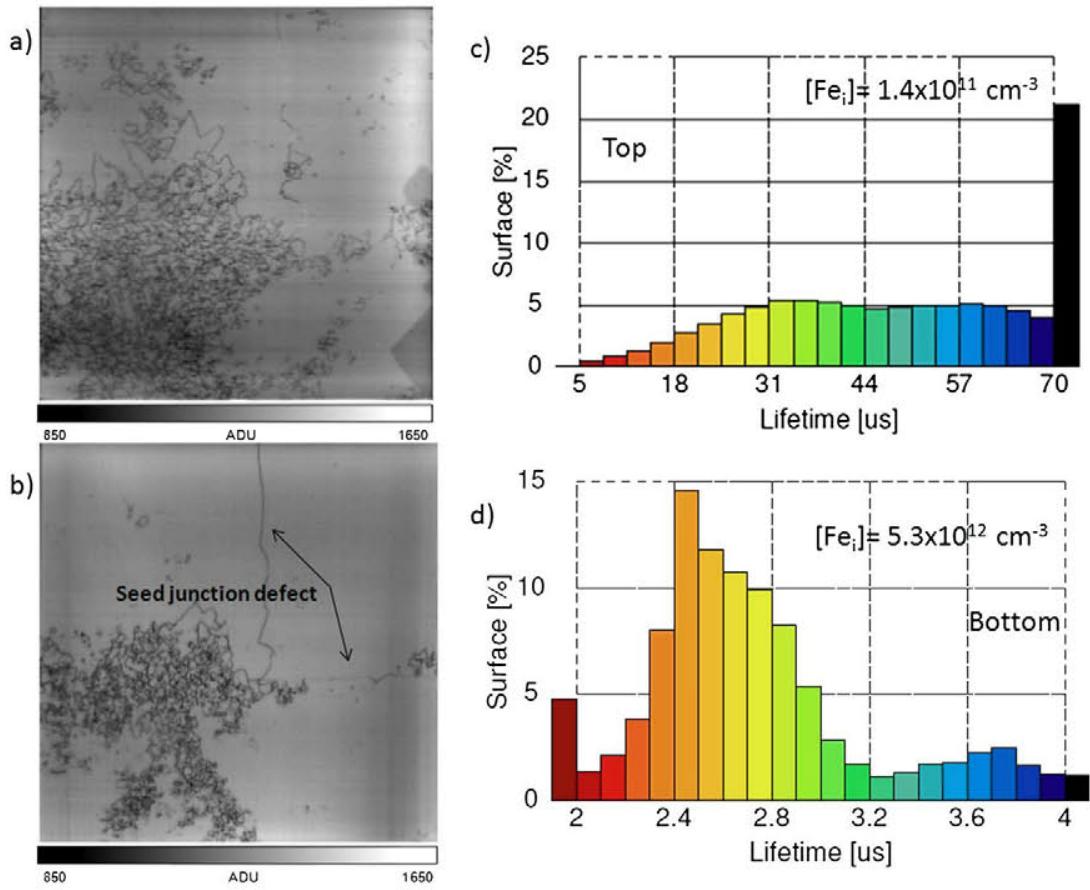

Figure 4. (a) Photoluminiscence image of an as-cut wafer from the top of the brick; (b) same analysis corresponding to a wafer from a lower region; (c) carrier lifetime histogram and [Fe] values of a wafer from the top of the brick; and (d) same analysis, wafer from the brick's bottom.

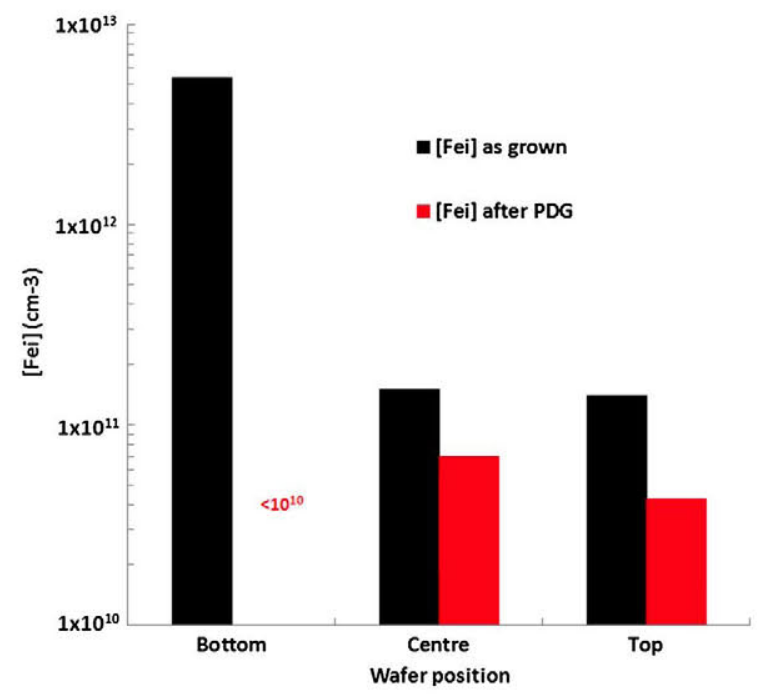

Figure 5. (a) Evolution of the $\left[\mathrm{Fe}_{\mathrm{j}}\right]$ before and after phosphorus gettering for wafers from bottom to top of a mono-like brick (1.1 $\Omega$-cm average resistivity).

even more marked than in a typical mc-Si cast growth (Figure 6(a)), which can have an important influence on the yield and mass production of this particular type of ingots.

In addition, a higher lateral curvature is evident when implementing the seed-cast growth approach in a typical DSS casting furnace (Figure 6(b)), in comparison with a common mc-Si process (Figure 6(c)). This is a consequence of the higher thermal gradients occurring during the growth, because the thermal history of the silicon mass differs from each other's type of ingot growth approach (Table I), due to the aforementioned passive heat extraction from the bottom of the crucible. Figure 7 represents two detrimental phenomena occurring in a brick squared from the ingot corner, from bottom to top, measured by PL: (a) increase of the mc-Si patterns towards the center of the brick; and (b) propagation of extended defects in the monocrystalline region of the wafer.

\subsection{Towards the seed recycling}

The use of high-quality $\mathrm{Cz}-\mathrm{Si}$ monocrystalline seeds is definitely critical for the obtention of high performance mono-like wafers. From an industrial standpoint, the implementation of this new growth approach at mass scale requires a detailed cost analysis and optimization to obtain profitability. A critical issue of the process refers to the manufacture and treatment of $\mathrm{Cz}-\mathrm{Si}$ ingots to make monocrystalline seeds, which can play a major role on both the cost per wafer and the cost per Watt. Therefore, the recycling of the virgin seeds (from a G0 process) seems mandatory. From the results presented before, the bottom section of the ingots, including the seed part, appears to be significantly affected after the first ingot growth run. Both crystal and impurity effects were noticed. Therefore, the question at this point is to know how much the quality of the seed is degraded in that region and what can be the consequences in the performance of wafers from $\mathrm{Gn}$ ingot growth runs. 

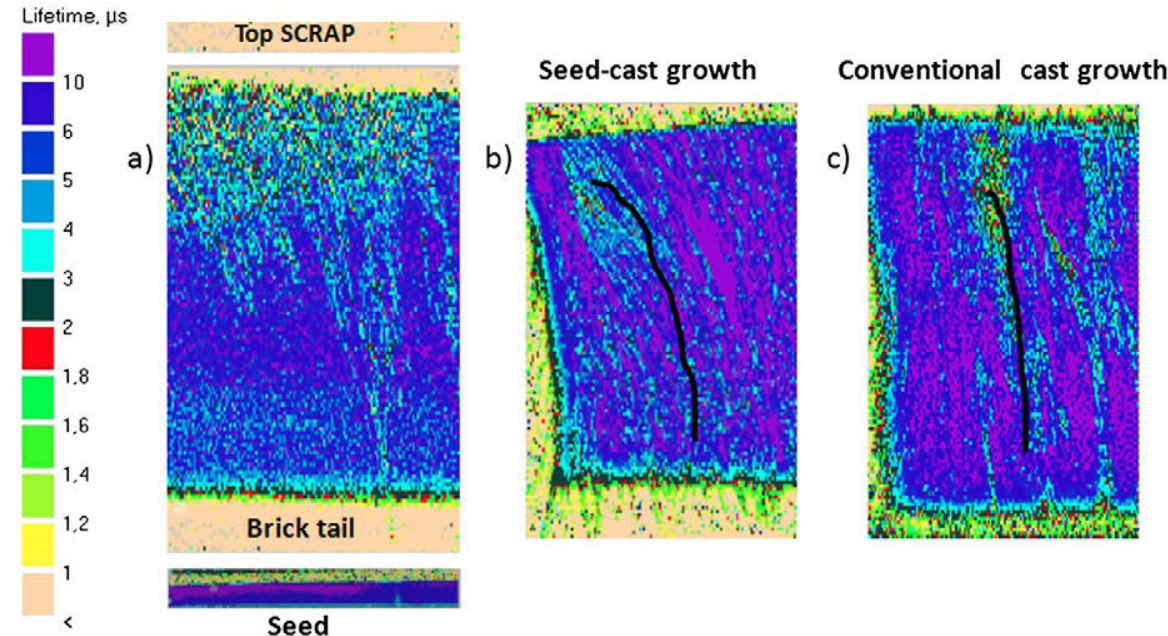

Figure 6. (a) Microwave photoconductance decay lifetime mapping of a brick (central) from a seed-cast growth process; (b) cropped brick (lateral) from the same ingot; and (c) cropped brick (lateral) from a common mc-Si cast ingot.

Table I. Comparison between thermal gradients for a seed-cast and a normal cast processes at three different stages of the crystal growth, at both the center and the lateral sides of the ingots, calculated from experimental data.

\begin{tabular}{lccccc}
\hline \multicolumn{3}{c}{ Thermal gradient K/cm } \\
\hline Interface position & \multicolumn{2}{c}{ Seed-cast } & & \multicolumn{2}{c}{ Normal cast } \\
\cline { 2 - 3 } \cline { 6 - 7 } & Center & Lateral & & Center & Lateral \\
\cline { 2 - 3 } & 15,1 & 10,4 & & 12,9 & 9,8 \\
$12 \mathrm{~cm}$ & 12,6 & 10,3 & & 11,5 & 9,1 \\
$24 \mathrm{~cm}$ & 8,8 & 7,1 & & 8,3 & 6,8 \\
\hline
\end{tabular}

For that purpose, a series of industrial size ingots were manufactured, monitoring the transverse $\mu$-PCD lifetime degradation of the seeds after consecutive seed-cast crystalline growths. Figure 8 illustrates an example, in which the analysis of half an ingot section-after a G0 process (degradation of the individual seeds after one mono-like process), then of G1 seeds to be used for the next growth run-collecting data from every respective seed profile. The figure includes also the lifetime map of one of the virgin seeds used to make the mono-like ingot. The degradation after the ingot growth process increases significantly, according to the following degradation features:

(i) Lifetime degradation towards the crucible base. Both solid-state diffusion of metal impurities and high oxygen concentration from the crucible are likely to be the causes of such a quality degradation.

(ii) Curved melting front of the seed. Both the heat exchange paths offered by the graphite's hot zone and the used furnace settings during the seed-cast growth result in a non-linear melting behavior of the seeds near the crucible walls. This is critical for recycling the seed, as the consecutive Gn slabs will contain larger mc-Si patterns, which will be transferred from the very beginning of the process. Also, these mc-Si grains propagate to the center of the ingot from bottom to top, as stated before (Figure 7).

(iii) Low lifetime region at the top of the seed. The larger the fraction of the melted seed, the deeper the low lifetime region after crystallization. Also, the back-diffusion of $\mathrm{Fe}$, which can promote the generation of crystal defects, is increased with seed recycling.

(iv) Low lifetime seed junctions. The respective edges of the seeds act as defect nucleation regions. In addition, the silicon melted mass over the slabs, presumably contaminated by $\mathrm{Fe}$, just prior to the crystal growth beginning, can percolate through those geometric imperfections, degrading those transition zones after solidification. This phenomenon would be also related to the degradation of the seed region in contact with the crucible base.

Consecutive recycling of the seeds can result in important loss of wafer electronic quality by $>0.7 \%$ in absolute efficiency. This is mainly caused by the remarkable decrease in the $I_{\mathrm{sc}}$ values, as the density of extended bulk defects dramatically increases. For a solar cell manufacture, the use of wafer alkaline texturing processes could mitigate to a certain extent such an adverse outcome, in terms of cost production balance issues (fair cost/W ratio). Figure 9(a) illustrates a PL analysis on wafers sliced from a G2 ingot. The image shows a mono-like wafer crisscrossed by dark line defects over the full surface, which directly results in poor $I-V$ solar cell performances. A simple surface cleaning by $\mathrm{KOH} / \mathrm{HCl}$ etch agents reveals the presence of crystal defects, such as subgrain boundaries [4] (Figure 9(b)). Moreover, a reduction of the percentage of the mono-like 


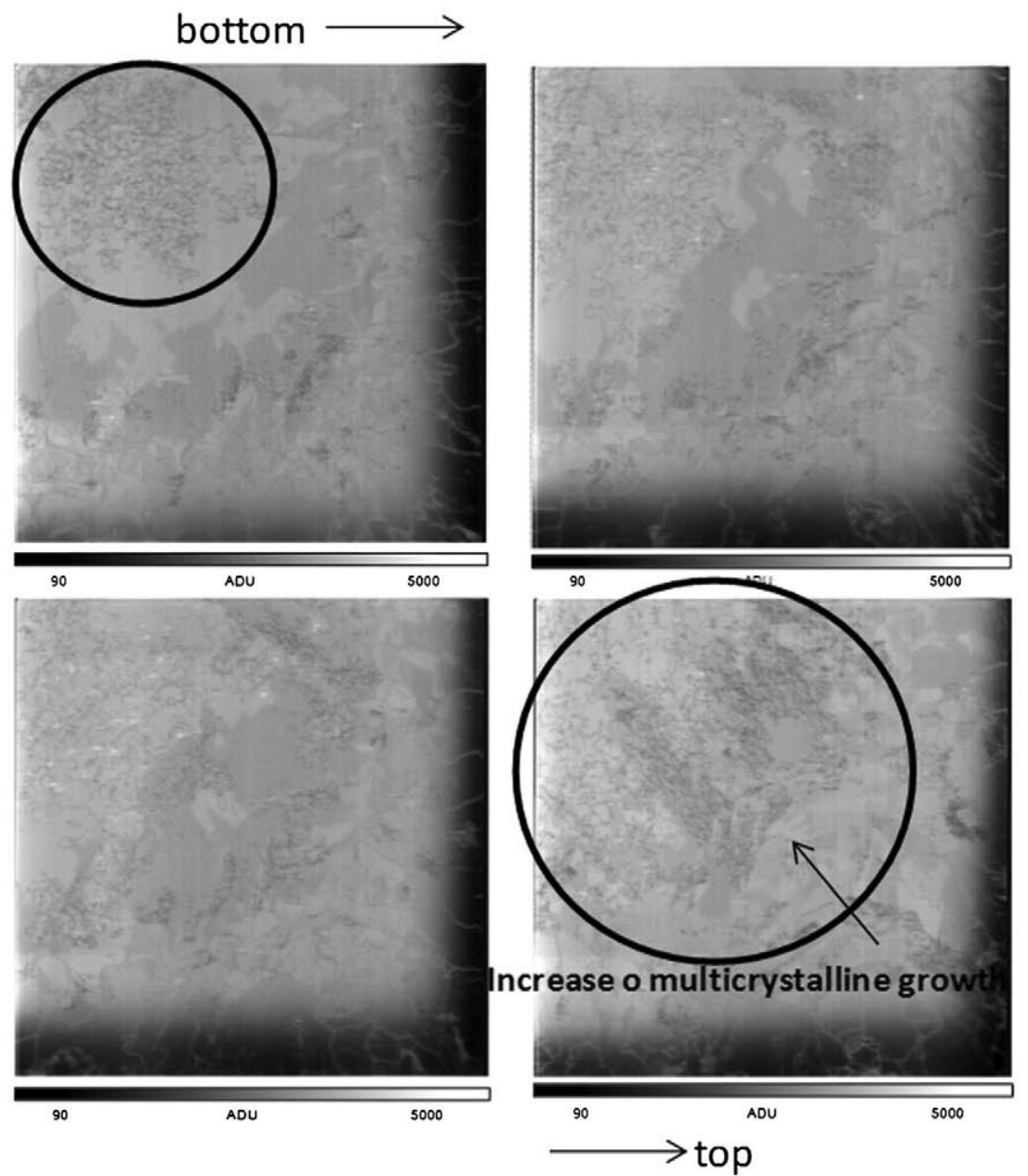

Figure 7. Photoluminiscence (PL) images of as-cut wafers of a corner brick, squared from a seed-cast ingot. The arrow indicates the mc-Si grown region increase, whereas the circles count on the density of extended defects growing from bottom to top, at the monocrystalline region of the wafer. The low activity dark regions at the right-down sides of the PL images are due to contact between silicon and the crucible walls, typically more contaminated from solid-state diffusion of impurities.

wafer yield per ingot can also be noted. Therefore, a mass production implementation of the seed-cast growth approach needs a dedicated and cost balanced technical revision.

The typical thermal stresses appearing during the silicon growth process, which result in plastic deformation leading to networks of dislocations, are suggested to be more pronounced from bottom to top. The occurrence of microdefects due to impurities precipitation, and $\mathrm{SiC}$ and $\mathrm{Si}_{3} \mathrm{~N}_{4}$ inclusions is also present in a seed-cast growth process, as it happens in a typical mc-Si casting process. As known, the presence of bulk defects can influence the mechanical properties of the wafers. Therefore, an increase in the macroscopic breakage yield during the industrial handling can be expected. It has been verified that there were direct effects of such a high density of defects (Figure 9(a)) in the fracture tension limits of the wafers, as determined by a systematic four-line bending test (FLBT) analysis [10]. By FLBT fitted to the corresponding Weibull distribution analysis, a batch of 50 highly dislocated mono-like wafers exhibited up to ca. $15 \%$ lower fracture tension average values than an equivalent number of samples of dislocation-free $\mathrm{Cz}$-Si square monocrystalline wafers, showing in any case a reasonable tension fracture behavior. These wafers were chemically etched to remove the surface damage generated during the sawing process, in order to highlight the intrinsic mechanical properties of the wafers.

The strain field of dislocations influences its recombination activity [11]. Consequently, additional studies on the electrical performance of these defects were performed using high spatial resolution LBIC analysis (better than $1 \mu \mathrm{m}$ ) on solar cells manufactured from different Gn-based wafers. LBIC maps (Figure 10) illustrate different charge capture activities from extended defects of neighboring regions, in the monocrystalline region of the wafer. Indeed, the electrical activity of such defects was found to be dependent on the Gn ingot growth, which would imply both an increase of the density of decorating defects and higher precipitated 


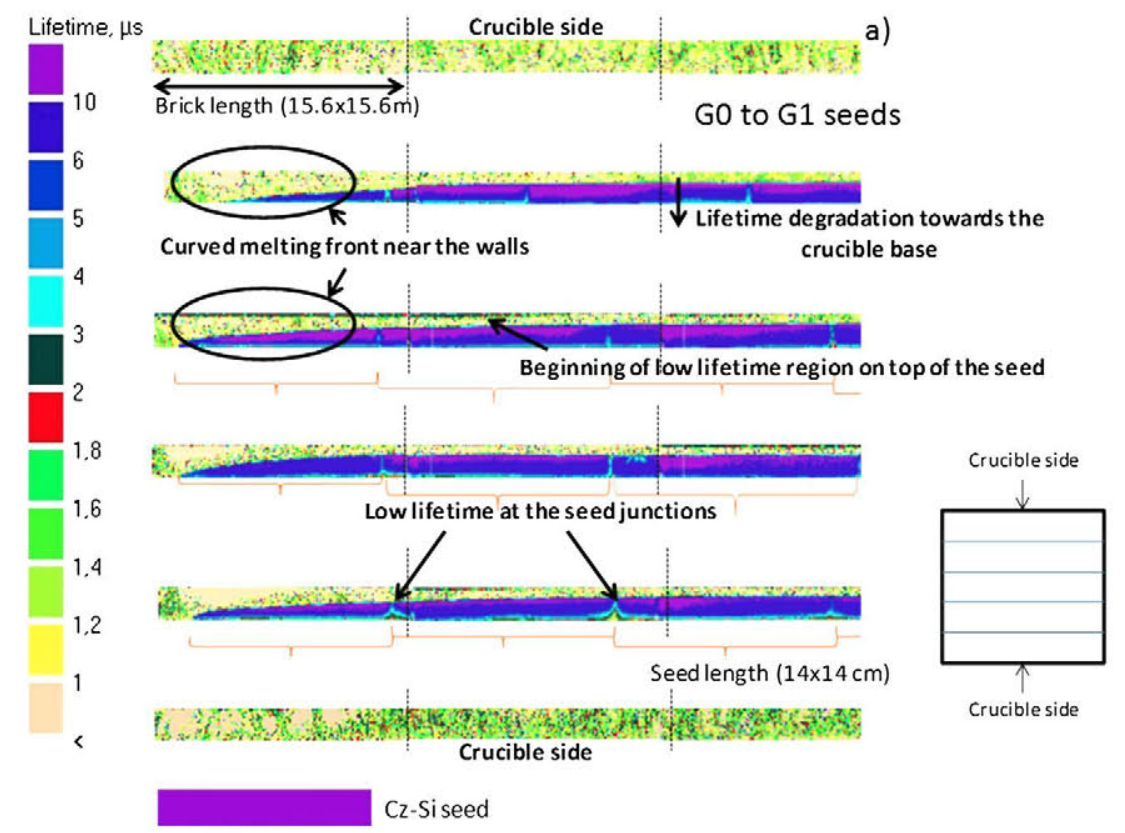

Figure 8. (a) Transversal microwave photoconductance decay lifetime mappings for a series of cropped seeds $(14 \mathrm{~cm} \times 14 \mathrm{~cm} \times 2 \mathrm{~cm}$ size) after having been used in the same G0 ingot growth (after just one seed-cast process). The scheme represents the section of half a directional solidification ingot ( 25 bricks $15.6 \mathrm{~cm} \times 15.6 \mathrm{~cm}$ size). A sketched chart of the top of an ingot is included as an inset, in order to clarify the cross sections of the seeds illustrated on the left side of the figure; (b) lifetime mappings of the same seeds after a second ingot growth (G1 process), in this case showing the entire $5 \times 5$ seeded ingot. Therefore, the more the number of seed recycling processes, the larger the lifetime degradation occurs in the slabs.

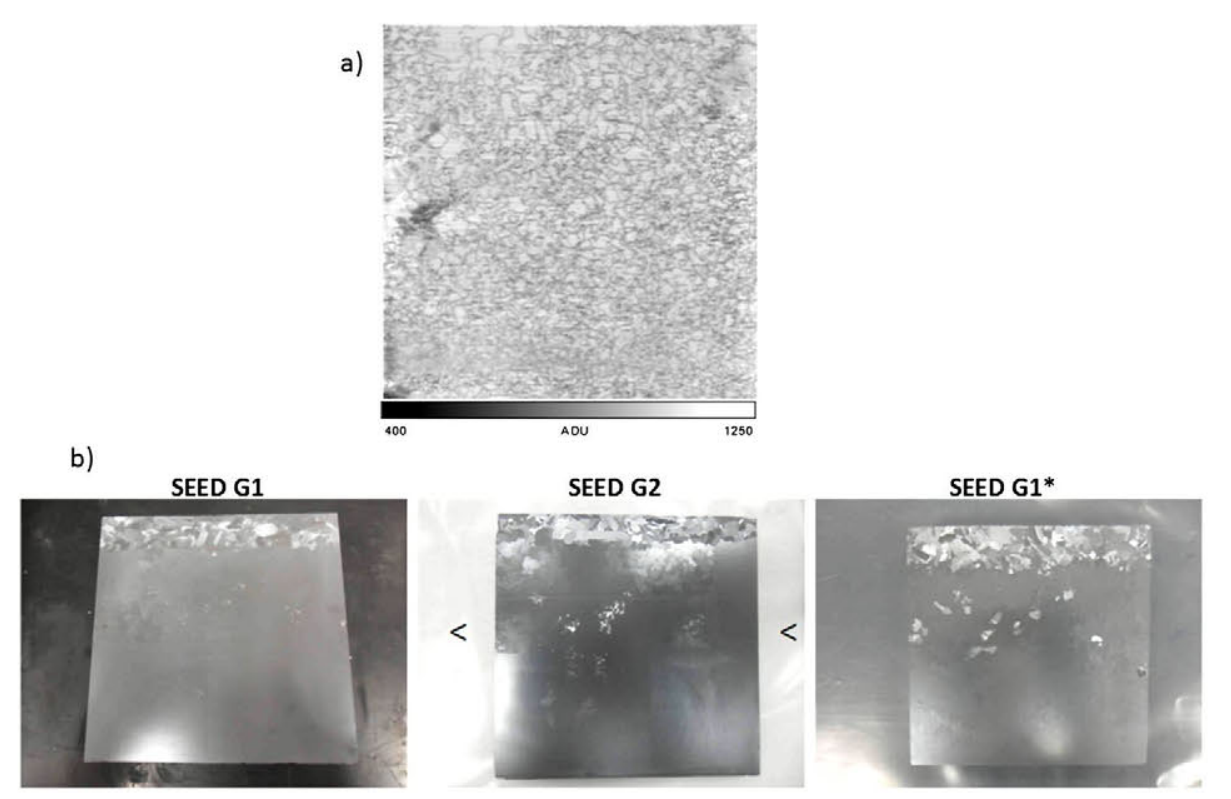

Figure 9. (a) Photoluminiscence image of a full mono-like wafer $(15.6 \mathrm{~cm} \times 15.6 \mathrm{~cm})$ coming from a $\mathrm{Gn}$ ingot, characterized by a large density of defects; (b) pictures of different seeds coming from the same lateral brick. G1* corresponds to a silicon slab used as mono-like seed, extracted from the immediate section above the G1 seed. Its crystal quality is rather poorer than that of a G2 seed.

impurities, in agreement with the loss of solar cell efficiency when increasing the number of seed recycling processes.
All the previously mentioned seed degradation sources still indicate that the recycling and/or regeneration of the 


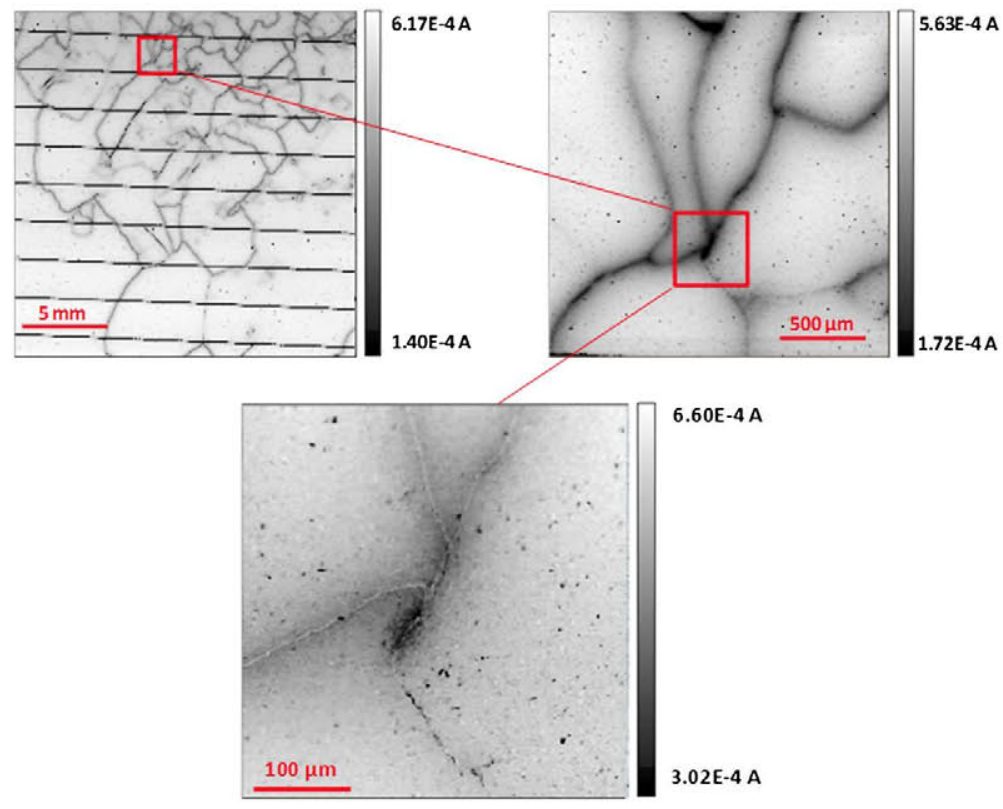

Figure 10. Laser beam induced current maps $(830 \mathrm{~nm}$ light excitation) of a solar cell made from a G1 ingot wafer. The three images represent different spatial resolutions. Note how the electrical activity of the intragrain defects appears non-homogeneous as the resolution is enhanced, which is the consequence of the impurity atmosphere surrounding the dislocations. In the largest resolution image, one clearly observes a bright contrast line drawing the defect and the dark atmosphere (charge capture) surrounding it.

original Cz-based silicon slab, even if highly recommended at industrial level, is not a straightforward task. However, both the quality devaluation effects produced on the seeds after consecutive mono-like ingot growth runs, and the extended defects generation during crystal growth can be alleviated to some extent. This can be achieved by designing and conducting a series of process optimizations and hardware modifications. The improving strategies can be listed as follows:

(i) Progressive melting of the seed during consecutive growth cycles, starting from an optimized silicon slab thickness. A perfect control of the melting front just before the initiation of the crystal growth is also recommended.

(ii) Promotion of a preferential heat flux along the seed versus the crucible sides, in order to avoid high thermal stresses that can generate high dislocation densities and decrease the mc-Si lateral pattern formation. This should be achieved by modifying the graphite susceptor design and properties and/ or the graphite block constituting the crucible base. Also, the crucible thickness can play a role, as it is formed by low thermal conductive fused silica.

(iii) In case of using a series of small size $\mathrm{Cz}-\mathrm{Si}$ seeds to promote the monocrystalline growth, and not only one large crystalline silicon slab located at the bottom of the crucible, a high-quality sawing process of the original seeds and the subsequent recycled fragments shall be performed. The control of the silicon orientation at the seeds cross sections should be taken into account, in order to get more stable crystalline boundaries between seeds.

(iv) Use low Fe contaminated crucible/inner coatings.

(v) Use different seed orientations to support the mono-like outcome all over the ingot, mainly at regions near the crucible sides and corners.

(vi) At solar cell level, a defined optimization of the texture processes for the non-perfect monocrystalline quality of the mono-like wafers would help to increase the cost/performance ratio, balancing the production costs over all the PV silicon chain.

\section{CONCLUSIONS}

The PV industry needs to innovate in order to acquire a sufficient robustness to approach and face the market fluctuations and the worldwide crisis period. In this sense, the implementation of the novel seed-cast growth can entail a realistic and feasible turning point for that industry, as the obtention of high device efficiency at optimized production costs seems possible, using common multicrystalline cast furnaces, which means that one does not need to tackle significant investments in new manufacturing infrastructure for reaching a substantial improvement of the Si based solar cell efficiency.

However, the seed-cast growth process still needs to be deeply reviewed before moving to mass scale activities, as a standard and stable industrial process. The sources of high defect generation, low performance ingot regions and the potential problems to reusing the original seed 
(necessary for cost reduction) in subsequent crystal growth runs have been discussed on the basis of the results obtained from a series of industrial scale experiments using typical casting furnace stations. Finally, in view of these considerations, several strategies have been proposed to improve the mono-like ingot performance and yield.

\section{ACKNOWLEDGEMENTS}

Funds from "Subprograma INNPACTO" (MICINN) IPT-420000-2010-22 project are gratefully acknowledged. Authors also appreciate the collaboration of SEMLAB (Hungary) for the PL image analysis. Dr. Nick Bateman and his team at Varian Semiconductor (Applied Materials, USA) are also acknowledged for the ion implantation cell manufacturing.

\section{REFERENCES}

1. Schmidt J, Bothe K. Structure and transformation of the metastable boron- and oxygen-related defect center in crystalline silicon. Physical Review B 2004; 69(2): 1-8.

2. Schmid F. Casting large silicon single-crystals. Journal of Electronic Materials 1976; 5(4): 436-437.

3. Stoddard N, Wu B, Witting I, Wagener M, Park Y, Rozgonyi G, Clark R. Casting single crystal silicon: novel defect profiles from BP Solar's Mono $2^{\mathrm{TM}}$ wafers. Solid State Phenomena 2008; 131-133: 1-8.

4. Jouini A, Ponthenier D, Lignier H, Enjalbert N, Marie B, Drevet B, Pihan E, Cayron C, Lafford T, Camel D. Improved mc-Si ingots crystal quality through seed growth for high efficiency solar cells. Progress in Photovoltaics: Research and Applications 2011. DOI: 10.1002/pip.1221
5. Gao B, Nakano S, Kakimoto K. Influence of backdiffusion of iron impurity on lifetime distribution near the seed-cast interface in seed-cast-grown monocrystalline silicon by numerical modeling. Crystal Growth \& Design 2012; 12:522-525.

6. Gao B, Nakano S, Harada H, Miyamura Y, Sekiguchi T, Kakimoto K. Reduction of polycrystalline grains region near the crucible wall during seeded growth of monocrystalline silicon in a unidirectional solidification furnace. Journal of Crystal Growth 2011. DOI: 10.1016/ j.jcrysgro.2011.11.084

7. Moralejo B, Hortelano V, González MA, Martínez O, Jiménez J, Ponce-Alcántara, S, Parra V. Study of the crystal features of mc-Si PV cells by laser beam induced current (LBIC). Physica Status Solidi C 2011; 8(4): 1330-1333.

8. Kvande R, Geerligs LJ, Coletti G, Arnberg L, Di Sabatino M, Øvrelid EJ, Swanson CC. Distribution of iron in multicrystalline silicon ingots. Joumal of Applied Physics 2008; 104: 064905.

9. Boulfrad Y, Stokkan G, M'Hamdi M, Øvrelid E, Arnberg L. Modeling of lifetime distribution in a multicrystalline silicon ingot. Solid State Phenomena 2011; 178-179: 507.

10. Barredo J, Parra V, Guerrero I, Fraile A, Hermanns L. On the mechanical strength of mono-, multi- and quasimono crystalline silicon wafers: a four-line bending test study. Progress in Photovoltaics: Research and Applications 2012. Submitted.

11. Ganapati V, Schoenfelder S, Castellanos S, Oener S, Koepge R, Sampson A, Marcus MA, Lai B, Morhenn H, Hahn G, Bagdahn J, Buonassisi T. Infrared birefringence imaging of residual stress and bulk defects in multicrystalline silicon. Journal of Applied Physics 2010; 108(6): 063528. 